\title{
Susceptibilidad antimicrobiana y diseminación policlonal de cepas de Staphylococcus aureus
}

\section{Maribel J. Castellano, Armindo J. Perozo, Ana M. Parra, Messaria M. Ginestre y Gresleida C. Rincón}

\section{Antimicrobial susceptibility and polyclonal dissemination of Staphylococcus aureus strains}

Objectives: To determine the prevalence of methicillin-resistant Staphylococcus aureus (MRSA), its antimicrobial susceptibility patterns and classify strains by pulsed-field gel electrophoresis (PFGE). Material and Methods: 106 $S$. aureus strains isolated from patients hospitalized at the Maracaibo city university hospital, Venezuela, were processed during the first quarter of 2009. The culture, isolation and identification of $S$. aureus were done by conventional methods. Antimicrobial susceptibility was determined by the disk diffusion method. The presence of $m e c A$ gene in MRSA strains was verified using the polymerase chain reaction (PCR). Results: Fifty-four strains $(50.94 \%)$ were MRSA and twenty three antibiotypes were detected. The most frequently observed was the one including $\beta$-lactams, macrolides, lincosamides, aminoglycosides and quinolones. There were forty multi-resistant isolates (74.0\%) between MRSA strains. All methicillin-resistant isolates were mecA positive. PFGE classified MRSA stains in 50 pulsotypes, each one containing between six and thirteen bands. Four small groups, of two strains each, had $80 \%$ of similarity. Five of the eight strains in these small clusters $(62.50 \%)$ had the same pattern of resistance. Conclusion: There is a high prevalence of multi-resistant MRSA strains with polyclonal dissemination in the hospital.

Key words: Staphylococcus aureus, methicillin, resistance, pulsed-field gel electrophoresis.

Palabras clave: Staphylococcus aureus, meticilina, resistencia, electroforesis en gel de campo pulsado.

\section{Introducción}

$\mathrm{M}$ undialmente, Staphylococcus aureus resistente a meticilina (SARM) se ha convertido en el patógeno intrahospitalario más significativo, siendo capaz de producir un amplio rango de infecciones y síndromes clínicos asociados con enfermedades graves, como bacteriemia, neumonía, endocarditis, artritis séptica, osteomielitis y la formación de abscesos profundos, entre otros $^{1}$. Debido a su gran capacidad de diseminación, es necesario implementar programas efectivos de vigilancia para la identificación y control de cepas epidémicas ${ }^{1,2}$. SARM suele ser resistente a todas las penicilinas, carbapenémicos y cefalosporinas. Los agentes antimicrobianos con actividad anti-SARM varían entre hospitales, ya que cada centro de salud puede tener su propio patrón de susceptibilidad. El conocimiento de la relación clonal de SARM y sus patrones de susceptibilidad antimicrobiana puede contribuir a los esfuerzos de control de infecciones hospitalarias en la supervisión y limitación de su propagación al interior de y entre hospitales ${ }^{2,3}$.

Sin dudas, en los últimos años, el amplio uso de antimicrobianos ha acelerado la evolución de $S$. aureus y conducido a la aparición de cepas que han adquirido sistemáticamente múltiples determinantes genéticos de resistencia ${ }^{4}$. Con el surgimiento de estos aislados multi-resistentes en los hospitales por un lado y, por el otro, la diseminación a la comunidad de cepas hipervirulentas de SARM, este microorganismo ha evolucionado muy rápidamente $\mathrm{y}$ ha creado nuevos problemas clínicos ${ }^{2,4}$.

Una de las formas eficientes para estudiar la propagación de SARM es a través de la determinación de las características genotípicas de los clones, así como el parentesco genético de las cepas en diferentes regiones geográficas $^{2,5-7}$. En los últimos años, se han utilizado una variedad de técnicas moleculares para la tipificación de las cepas de $\mathrm{SARM}^{8}$. De éstas, el análisis de macro-restricción mediante electroforesis en gel de campo pulsado (EGCP), utilizando la enzima SmaI es el "estándar de oro" para el análisis de la epidemiología local a corto plazo ${ }^{6,9,10}$.

Hasta hace poco se afirmaba que la diseminación de SARM era consecuencia de la propagación global de pocos clones altamente epidémicos ${ }^{11}$; sin embargo, estudios recientes han mostrado evidencias de que la población de SARM está geográficamente estructurada y que puede haber surgido frecuentemente, en diferentes partes del mundo, gracias a incorporaciones independientes de determinantes de resistencia a meticilina en su genoma ${ }^{2,12}$. En consecuencia, para comprender mejor la emergencia y difusión global de SARM es útil obtener información acerca de la epidemiología local. Lamentablemente, hasta la fecha, los trabajos en relación
Escuela de Bioanálisis. LUZ. Maracaibo-Venezuela.

Cátedra de Bacteriología General (MJC, MMG).

Cátedra de Bacteriología Clínica (GCR).

Centro de Referencia Bacteriológica SAHUM. Maracaibo-Venezuela. Cátedra de Práctica Profesional de Bacteriología (AJP, (AMP)

Este proyecto fue financiado por el Consejo de Desarrollo Científico y Humanístico de la Universidad del Zulia (Proyectos No VAC-CONDESCC-0733-08 y VAC-CONDES -CC-0065-12).

Los autores no declaran conflictos de interés.

Recibido: 22 de mayo de 2013 Aceptado: 2 de enero de 2014

Correspondencia a: Maribel J. Castellano maribeljo@cantv.net. 
a la epidemiología molecular de SARM en el área son muy escasos.

Obedeciendo a esta inquietud, se realizó la presente investigación cuyos objetivos fueron: determinar la prevalencia de SARM; determinar los perfiles de susceptibilidad antimicrobiana en cepas de $S$. aureus aisladas de pacientes hospitalizados y tipificar las cepas mediante EGCP para establecer el posible origen clonal de las cepas de SARM, información relevante para el personal de salud y los comités de vigilancia y control de las infecciones intrahospitalarias para implementar los programas y estrategias de intervención en salud.

\section{Materiales y Métodos}

\section{Cepas bacterianas}

Se estudiaron 106 cepas de S. aureus aisladas de diferentes muestras clínicas provenientes de pacientes internados en un hospital universitario de la ciudad de Maracaibo, Venezuela, durante el primer trimestre del año 2009. El cultivo, aislamiento e identificación bacteriana se efectuó acorde a la metodología convencional. De todo paciente incluido en esta investigación se obtuvo el consentimiento informado por escrito. En el caso de los menores de edad, se solicitó la autorización de sus padres o representantes. El nombre se mantuvo en el anonimato para garantizar la privacidad de los pacientes. Todos los procedimientos se realizaron de acuerdo a las normas establecidas por la Organización Mundial de la Salud (OMS) y el Código de Bioseguridad y Bioética de la República Bolivariana de Venezuela en su tercera edición ${ }^{13}$. Se contó además, con la autorización del Comité de Ética del hospital.

\section{Determinación de los fenotipos de resistencia antimicrobiana}

Las pruebas de susceptibilidad antimicrobiana fueron realizadas fenotípicamente mediante el método de difusión con disco en agar, siguiendo las directrices del Instituto para la Estandarización de Laboratorios Clínicos $(\mathrm{CLSI})^{14}$. Los discos de antimicrobianos probados fueron: $\beta$-lactámicos: penicilina $\mathrm{G}(10 \mathrm{U})$; oxacilina $(\mathrm{OX}, 1 \mu \mathrm{g})$; y cefoxitin (FOX, $30 \mu \mathrm{g})$; aminoglucósidos: amikacina $(\mathrm{AK}, 30 \mu \mathrm{g})$ y gentamicina (GM, $10 \mu \mathrm{g})$; quinolonas: ciprofloxacina (CIP, $5 \mu \mathrm{g}$ ); gatifloxacina (GAT, $5 \mu \mathrm{g}$ ) y levofloxacina (LEV, $5 \mu \mathrm{g}$ ); diaminopirimidinas: cotrimoxazol (STX, 1,25/23,75 $\mu \mathrm{g}$ ); cetólidos: telitromicina (TEL, $15 \mu \mathrm{g})$; lincosamidas: clindamicina (CC, $2 \mu \mathrm{g}$ ); macrólidos: eritromicina (E, $15 \mu \mathrm{g})$; estreptograminas: quinupristin/dalfopristin (QDA, $15 \mu \mathrm{g})$; oxazolidinonas: linezolid (LZD, $30 \mu \mathrm{g}$ ); glicopéptidos: teicoplanina (TEC, $30 \mu \mathrm{g})$; fenicoles: cloranfenicol (C, $30 \mu \mathrm{g})$; tetraciclinas: tetraciclina (TE, $30 \mu \mathrm{g})$; rifamicinas: rifampicina (RA,
$5 \mu \mathrm{g})$; glicilglicinas: tigeciclina (TGC, $15 \mu \mathrm{g})$; otros: fosfomicina (FOS, $50 \mu \mathrm{g}$ ) y mupirocina (MUP, $5 \mu \mathrm{g}$ ). Para la interpretación de los resultados de FOS, se utilizaron los criterios establecidos por Gobernado ${ }^{15} \mathrm{y}$ para MUP, los descritos por la Sociedad Británica para Quimioterapia Antimicrobiana (BSAC) ${ }^{16}$; para el resto de los antimicrobianos se utilizaron los criterios de interpretación del CLSI ${ }^{14}$. Para el control de calidad de las pruebas de susceptibilidad antimicrobiana, se utilizaron las cepas de $S$. aureus ATCC $^{\circledR} 25923$ (sensible a todos los antimicrobianos) y $S$. aureus ATCC $^{\circledR} 43300$ (resistente a oxacilina-meticilina).

Para la antibiotipia (determinación de los fenotipos de resistencia), cepas resistentes e intermedias fueron tratadas de manera indistinta. Aislados con resistencia, al menos, a un (1) agente antimicrobiano fueron considerados de un fenotipo distinto a aquellos completamente susceptibles.

Detección del gen mecA mediante la reacción de polimerasa en cadena (RPC): La extracción de ADN de colonias de $S$. aureus se llevó a cabo utilizando un procedimiento de lisis enzimática y la detección del gen $m e c A$ por RPC se efectuó de acuerdo a la metodología descrita por Martineau y cols ${ }^{17}$.

Tipificación de las cepas SARM por electroforesis en gel de campo pulsado: Para la tipificación molecular de las cepas SARM se utilizó el equipo GUEFAST $06^{\circledR}$ de Neuronic $^{\circledR}$, S.A. y para la obtención del ADN genómico de bacterias intacto e inmovilizado, se utilizó el BACKIT $\left(\text { Neuronic }^{\circledR}, \text { S. A }\right)^{18}$, acorde a las instrucciones del fabricante. Los perfiles obtenidos mediante la electroforesis fueron comparados mediante análisis computarizado, utilizando el software GUEFASCAN ${ }^{\circledR}$, provisto con el equipo.

\section{Análisis estadístico}

Para el registro de la información y la determinación de los perfiles de resistencia antimicrobiana se utilizó el programa Whonet ${ }^{\mathrm{TM}}$, versión 5,6 (World Health Organization). Para el estudio de la relación clonal se utilizó el programa SPSS para Windows ${ }^{\circledR}$, versión 20, realizando el análisis cluster jerárquico para clasificar las cepas de SARM estudiadas en grupos, de acuerdo a sus semejanzas y diferencias. Un cluster fue definido como un conjunto de patrones de bandas con más de $80 \%$ de similitud. Se utilizó una significancia del 5\% $(\alpha=0,05)$.

\section{Resultados}

La distribución de las cepas de $S$. aureus aisladas según el tipo de muestra se muestra en la Figura 1, encontrándose una mayor cantidad de cultivos positivos a partir de secreciones varias (52 cepas, 49,0\%); seguido de sangre 
y heridas quirúrgicas con 13 cepas en cada caso (12,2\%); aspirados traqueales con 12 cepas $(11,3 \%)$; abscesos con 8 cepas $(7,5 \%)$; catéteres centrales 3 cepas $(2,8 \%)$; lavados bronquiales 3 cepas $(2,8 \%)$ y, una cepa $(0,9 \%)$ para muestras de orina y exudado faríngeo, respectivamente. De las 106 cepas de $S$. aureus estudiadas, 54 (50,9\%) resultaron resistentes a meticilina, tanto con el disco de OX como con el de FOX y, 52 (49,0\%), sensibles a este antimicrobiano. Los porcentajes de resistencia antimicrobiana en cepas de $S$. aureus fueron: $97,1 \%$ de resistencia a PG (n: 103 cepas); 50,9\% a OX (n: 54); 43,3\% a E (n: 46); 39,6\% a TEL (n: 42); 35,8\% a CIP (n: 38); 34,9\% a CC (c: 37 ); $34,9 \%$ (n: 37) para cada uno de los aminoglucósidos ensayados (AK y GM); 29,2\% (n: 31) a LEV; 28,3\% a GAT (n: 30$) ; 9,4 \%$ (n: 10$)$ a TE; $1,8 \%$ a C (n: 2 cepas); $0,9 \%$ a RA (n: 1) e igual porcentaje para STX. Adicionalmente, se obtuvo $12,2 \%$ (n: 13 ) de cepas intermedias a E; 3,7\% (n: 4) a LEV y GAT; $1,8 \%$ (n: 2 ) a C y TE y $0,9 \%$ (n: 1$)$ a CIP y CC, respectivamente. No se observó resistencia para TEC, LZD, TGC, QDA, FOS y MUP.

La distribución de los porcentajes de resistencia a los antimicrobianos, según se tratara de cepas SARM o $S$. aureus sensible a meticilina (SASM), se muestra en la Figura 1 y Tabla 1. Las diferencias observadas entre los dos grupos de cepas estudiadas (SASM y SARM) fueron estadísticamente significativas $(\mathrm{p}<0,05)$ para $\mathrm{E}, \mathrm{CC}$, TEL, GM, AK, CIP, LEV y GAT; pero no para STX, TE, C y RA $(p>0,05)$. En el caso de LZD, TEC, QDA, FOS, MUP y FUS, no se pudo calcular el estadístico $\chi^{2}$ debido a que la susceptibilidad de las cepas fue una constante.

Los perfiles de resistencia acompañante determinados en las cepas SARM y SASM aparecen descritos en la Tabla 2. Entre las cepas SASM, la multi-resistencia alcanzó a 14 aislados (26,9\%), correspondiendo el mayor porcentaje $(15,3 \%)$ a ocho cepas resistentes a PG, E y TEL. En 9,6\% de los casos ( $n$ : 5 cepas) se detectó resistencia a cuatro grupos de antimicrobianos: PG, E, TEL y TE ó PG, E, TEL y CC. En una cepa que correspondió a 1,9\% de los casos, se demostró resistencia a cinco grupos de antimicrobianos: penicilinas, aminoglucósidos, macrólidos, lincosamidas y cetólidos. Cabe destacar que tres $(15,7 \%)$ de estas cepas fueron totalmente sensibles a los antimicrobianos probados y que la mayoría de las cepas SASM incluidas en este estudio $(53,8 \%)$ estuvo representada por aislados de $S$. aureus que sólo eran resistentes a PG (n: 28 cepas).

Entre las cepas SARM se observó mayor porcentaje de multi-resistencia (74,0\%; n: 40 aislados); de los cuales 30 $(55,5 \%)$ implicaban resistencia a cinco grupos de antimicrobianos: $\beta$-lactámicos, aminoglucósidos, lincosamidas, macrólidos y quinolonas y, cuatro $(7,4 \%)$ incluían los antimicrobianos anteriores más cloranfenicol. Cuatro cepas $(7,4 \%)$ expresaron resistencia a tres de los grupos de antimicrobianos probados; una $(1,8 \%)$ a cuatro grupos e igual cantidad a cinco de las clases de antimicrobianos

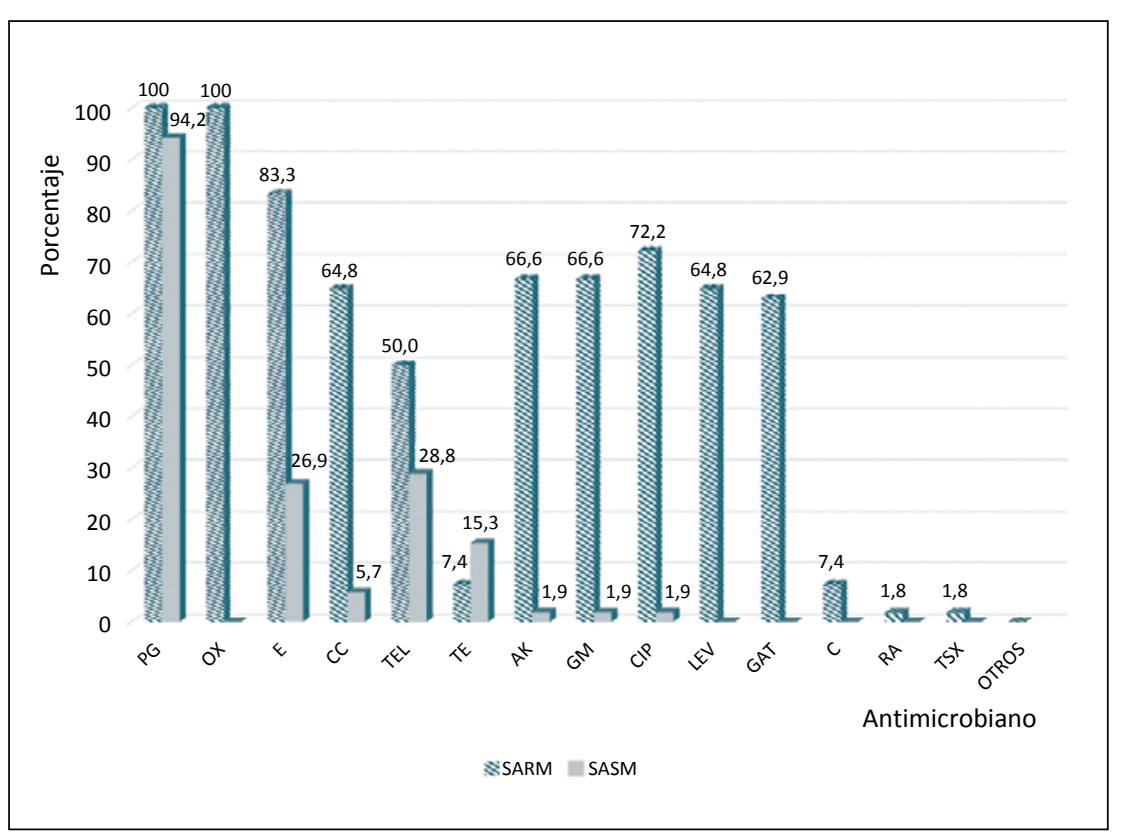

Figura 1. Distribución de los porcentajes de resistencia antimicrobiana en cepas intrahospitalarias de S. aureus de acuerdo a la susceptibilidad a meticilina. Maracaibo; Enero-Marzo 2012 ( $n=106$ ). Otros: TEC, TGC, QDA, LZD, FOS y MUP (abreviaturas en Material y Métodos).

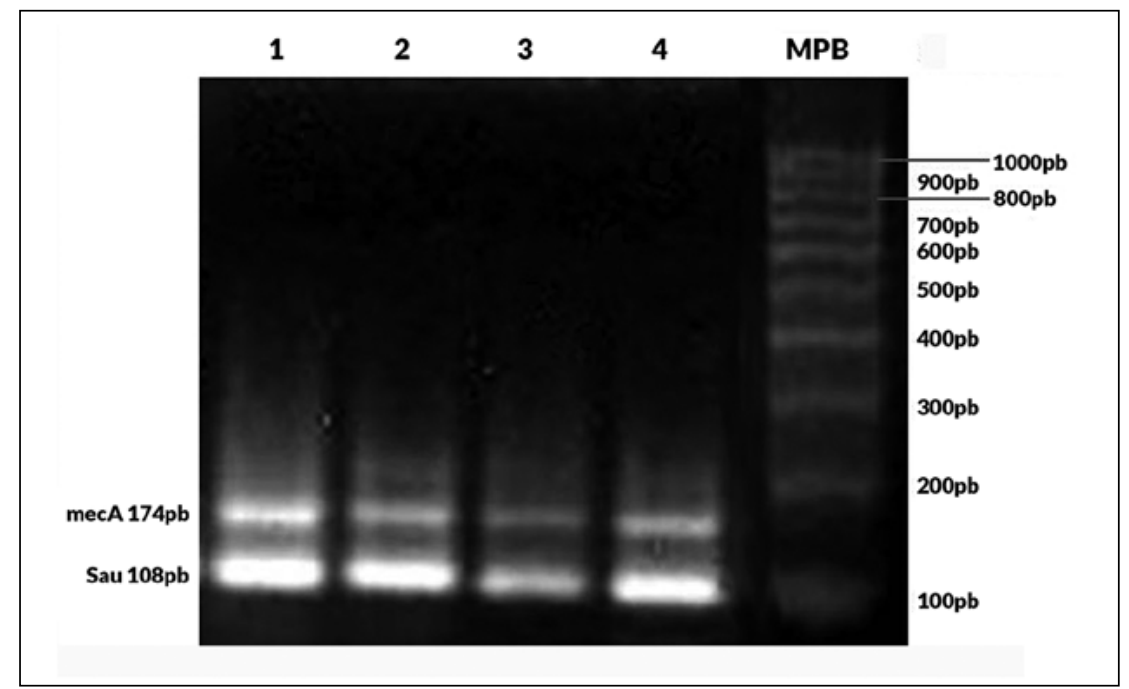

Figura 2. Resultado de la RPC para el gen mecA en cepas de $S$. aureus. Los carriles 1 al 4 muestran 2 bandas: una inferior que se refiere al control interno para la identificación de $S$. aureus (108 pb), y una superior que corresponde a la amplificación del gen mecA (responsable de la resistencia a meticilina) (174 pb).

utilizados. En 11 aislados que representan el 20,3\% de las cepas de SARM, se encontró resistencia a dos grupos de antimicrobianos: $\beta$-lactámicos y quinolonas (CIP); $\beta$-lactámicos y macrólidos $(\mathrm{E})$; $\beta$-lactámicos y tetraciclinas (TE) y $\beta$-lactámicos y aminoglucósidos (GM) y en tres $(5,7 \%)$ sólo se observó resistencia a $\beta$-lactámicos. Las diferencias observadas entre los porcentajes de resistencia 


\begin{tabular}{|c|c|c|c|c|}
\hline Fenotipo & $\begin{array}{c}\text { Grupos de } \\
\text { antimicrobianos } \\
\text { a los que expresa } \\
\text { resistencia }\end{array}$ & $\begin{array}{l}\text { Antimicrobianos } \\
\text { a los que expresa } \\
\text { resistencia }\end{array}$ & $\begin{array}{l}\mathrm{n} \text { de } \\
\text { cepas }\end{array}$ & $\%$ \\
\hline 1 & 0 & Ninguno & 3 & 2,83 \\
\hline 2 & 1 & PG & 28 & 26,42 \\
\hline 3 & & PG-OX & 6 & 5,66 \\
\hline 4 & 2 & PG-E & 5 & 4,73 \\
\hline 5 & & PG-OX-E & 5 & 4,73 \\
\hline 6 & & PG-CIP & 1 & 0,94 \\
\hline 7 & & PG-OX-CIP & 1 & 0,94 \\
\hline 8 & & PG-OX-GM & 1 & 0,94 \\
\hline 9 & & PG-TEL & 1 & 0,94 \\
\hline 10 & & PG-OX-TE & 1 & 0,94 \\
\hline 11 & 3 & PG-E-TEL & 5 & 4,73 \\
\hline 12 & & PG-OX-TE-E & 2 & 1,89 \\
\hline 13 & & PG-OX-CC-E & 1 & 0,94 \\
\hline 14 & & PG-OX-E-AK & 1 & 0,94 \\
\hline 15 & & PG-OX-E-CIP-LEV & 1 & 0,94 \\
\hline 16 & 4 & PG-E-TEL-TE & 3 & 2,83 \\
\hline 17 & & PG-E-TEL-CIP & 3 & 2,83 \\
\hline 18 & & PG-E-TEL-CC & 1 & 0,94 \\
\hline 19 & & PG-E-TEL-C & 1 & 0,94 \\
\hline 20 & & PG-OX-TE-E-STX-RA & 1 & 0,94 \\
\hline 21 & 5 & PG-E-TEL-CC-AK-GM-CIP & 1 & 0,94 \\
\hline 22 & & $\begin{array}{c}\text { PG-OX-AK-GM-E-CC-CIP- } \\
\text { LEV-GAT }\end{array}$ & 30 & 28,30 \\
\hline 23 & 6 & $\begin{array}{c}\text { PG-OX-AK-GM-E-CC-CIP- } \\
\text { LEV-GAT-C }\end{array}$ & 4 & 3,77 \\
\hline
\end{tabular}

PG: penicilina G; OX: oxacilina; E: eritromicina; CIP: ciprofloxacina; GM: gentamicina; TEL: telitromicina; TE: tetraciclina; CC: clindamicina; AK: amikacina; LEV: levofloxacina; C: cloranfenicol; STX: cotrimoxazol; RA: rifampicina; GAT: gatifloxacina.

acompañante encontrados en las cepas de $S$. aureus están estadísticamente relacionadas con la susceptibilidad a meticilina $(\mathrm{p}<0,05)$.

En las $54(50,9 \%)$ cepas de $S$. aureus, que eran resistentes a oxacilina por los métodos fenotípicos se detectó el gen mecA (Figura 2). Las cepas $m e c A$ positivas amplificaron una banda de 174 bp correspondiente al gen determinante de la resistencia a $\mathrm{OX}$ y todas amplificaron el gen utilizado para verificar la identificación de $S$. aureus, evidenciado por la presencia de una banda a $108 \mathrm{bp}$. No se encontró cepa alguna mec $A$ positiva y fenotípicamente susceptible a oxacilina.

En el dendrograma (Figura 3), se evidencia la existencia de cuatro pequeños grupos, de dos cepas cada uno, que corresponden a cepas que presentan hasta $80 \%$ de similitud (coinciden con el punto 5 de
Tabla 2. Distribución de los perfiles de resistencia en cepas intrahospitalarias de $S$. aureus de acuerdo a meticilina. Maracaibo, enero-marzo $2012(n=106)$

\begin{tabular}{|c|c|c|c|c|c|}
\hline Cepas & Fenotipo & $\begin{array}{c}\text { Grupos de } \\
\text { antimicrobianos } \\
\text { a los que expresa } \\
\text { resistencia }\end{array}$ & $\begin{array}{l}\text { Antibióticos } \\
\text { a los que } \\
\text { expresa } \\
\text { resistencia }\end{array}$ & $\begin{array}{l}\mathrm{n} \text { de } \\
\text { cepas }\end{array}$ & $\%$ \\
\hline \multirow{12}{*}{ SARM } & 1 & 1 & PG-OX & 3 & 5,56 \\
\hline & 2 & 2 & PG-OX-CIP & 4 & 7,41 \\
\hline & 3 & & PG-OX-E & 5 & 9,26 \\
\hline & 4 & & PG-OX-TE & 1 & 1,85 \\
\hline & 5 & & PG-OX-GM & 1 & 1,85 \\
\hline & 6 & & PG-OX-E-CC & 1 & 1,85 \\
\hline & 7 & & PG-OX-E-TE & 2 & 3,70 \\
\hline & 8 & & PG-OX-E-AK & 1 & 1,85 \\
\hline & 9 & & PG-OX-E-CIP-LEV & 1 & 1,85 \\
\hline & 10 & & $\begin{array}{c}\text { PG-OX-TE-E-STX- } \\
\text { RA }\end{array}$ & 1 & 1,85 \\
\hline & 11 & & $\begin{array}{c}\text { PG-OX-E-CC-GM- } \\
\text { CIP-LEV-GAT }\end{array}$ & 30 & 55,56 \\
\hline & 12 & & $\begin{array}{l}\text { PG-OX-E-CC-AK- } \\
\text { GM-CIP-LEV-GAT-C }\end{array}$ & 4 & 7,41 \\
\hline \multirow{9}{*}{ SASM } & 1 & & Ninguno & 3 & 5,77 \\
\hline & 2 & & PG & 28 & 53,85 \\
\hline & 3 & & PG-TE & 5 & 9,62 \\
\hline & 4 & & PG-CIP & 1 & 1,92 \\
\hline & 5 & & PG-TEL & 1 & 1,92 \\
\hline & 6 & & PG-E-TEL & 8 & 15,38 \\
\hline & 7 & & PG-E-TEL-TE & 3 & 5,77 \\
\hline & 8 & & PG-E-CC-TEL & 2 & 3,85 \\
\hline & 9 & & PG-ECC-AK-GM-TEL & 1 & 1,92 \\
\hline
\end{tabular}

PG: penicilina G; OX: oxacilina; E: eritromicina; CIP: ciprofloxacina; GM: gentamicina; TEL: telitromicina; TE: tetraciclina; CC: clindamicina; AK: amikacina; LEV: levofloxacina; C: cloranfenicol; STX: cotrimoxazol; RA: rifampicina; GAT: gatifloxacina.

la escala); nueve grupos presentaron una homología de hasta 60\%; veintiún conglomerados tenían un $40 \%$ de semejanza, nueve mostraron una similitud de apenas $20 \%$ y tres representaban cepas, prácticamente, $100 \%$ diferentes entre ellas; observándose en total, cuarenta y seis conglomerados.

Mediante la EGCP, las 54 cepas de SARM analizadas se clasificaron en 50 pulsotipos según los patrones de corte obtenidos, conteniendo entre seis y diecisiete bandas. A cada pulsotipo se le asignó un número (1 al 50). A pesar que el número de cepas presentes en cada uno de los pequeños clusters con alto porcentaje de similitud (dos), no permite hacer estimaciones estadísticas, se evidencia al comparar los patrones de bandas de las cepas 40 y 49, que éstas se diferencian en una banda que está presente en la 40 que no se encuentra en la 49 y en una banda que está presente en la cepa 49, más no en la 40 (Figura 4).

Por su parte los pulsotipos correspondientes a las cepas 3 y 4 , difieren en dos bandas, una exclusiva de la 3 y una de la 4 . Las cepas identificadas con los números 22 y 27 se distinguen en dos bandas que posee la 27 y no la 22, y en la intensidad de todas las bandas. Entre las cepas 18 y 19 existe una banda distinta que sólo se encuentra en la 18 (Figura 4). 
La gran variedad de pulsotipos detectados mediante el campo pulsado y el amplio rango de conglomerados que se pueden conformar entre las cepas SAMR estudiadas demuestran que estos grupos no están relacionados ya que, aunque puedan corresponder a una línea evolutiva común, su relación genética no es muy cercana, y es menos probable su relación epidemiológica; por tanto, se infiere que pertenecen a clones distintos.

La relación entre los antibiotipos y pulsotipos encontrados en las cepas de SARM demostró que cinco de las ocho cepas presentes en los cuatro pequeños clones mostraron el mismo fenotipo de resistencia: aminoglucósidos, macrólidos, lincosamidas, cetólidos y quinolonas; mientras que las tres cepas restantes presentaron un espectro de resistencia más estrecho, afectando únicamente a los $\beta$-lactámicos; $\beta$-lactámicos, macrólidos y cetólidos $\mathrm{o}$; $\beta$-lactámicos, tetraciclinas y glicil-glicinas.

\section{Discusión}

Es bien conocido que la prevalencia de $S$. aureus y su variedad resistente a meticilina, varían de un área geográfica a otra ${ }^{19}$; sin embargo, los recursos para monitorear la epidemiología de SARM en Latinoamérica continúan siendo limitados ${ }^{20}$. Gracias al esfuerzo de la Organización Panamericana de la Salud ${ }^{21}$, se ha logrado establecer un amplio rango de prevalencias que van desde las bajas, correspondientes a: Cuba, Honduras, y Nicaragua $(6 \% ; 12 \%$ y $20 \%$, respectivamente); pasando por las moderadas, correspondientes a Ecuador (20\%), Venezuela (25\%); Bolivia (36\%); Argentina (42\%); Paraguay (44\%) y Colombia (47\%); hasta llegar a las más altas que se encuentran en: México (52\%); Costa Rica (58\%); Uruguay (59\%); Guatemala (64\%); Chile y Perú, con $80 \%$ cada uno ${ }^{22-27}$.

En nuestra localidad, estudios previos realizados por este equipo de investigación refieren un 17,5\% de prevalencia global para SARM en la institución, el $82 \%$ de estas cepas eran de pacientes hospitalizados ${ }^{10}$, resultados que difieren abiertamente con los obtenidos en el presente trabajo, donde se demuestra que la prevalencia de SARM, lejos de disminuir, a pesar del reforzamiento de las medidas de control, ha aumentado alcanzando el $50,9 \%$ (casi 34\% más); lo que refleja la problemática mundial con este patógeno, que cada vez se hace más prevalente en el ambiente hospitalario, constituyendo una gran amenaza para la salud pública, debido a su potencial diseminación a la comunidad.

En el presente estudio, todas los aislados de SARM provenientes de diferentes tipos de muestras portaban el gen $m e c A$; $\sin$ embargo, existen reportes de cepas que muestran resistencia heterogénea, de bajo nivel de resistencia a oxacilina mediada por $m e c A$; y que, frente a la exposición a $\beta$-lactámicos, pueden expresar esta re-

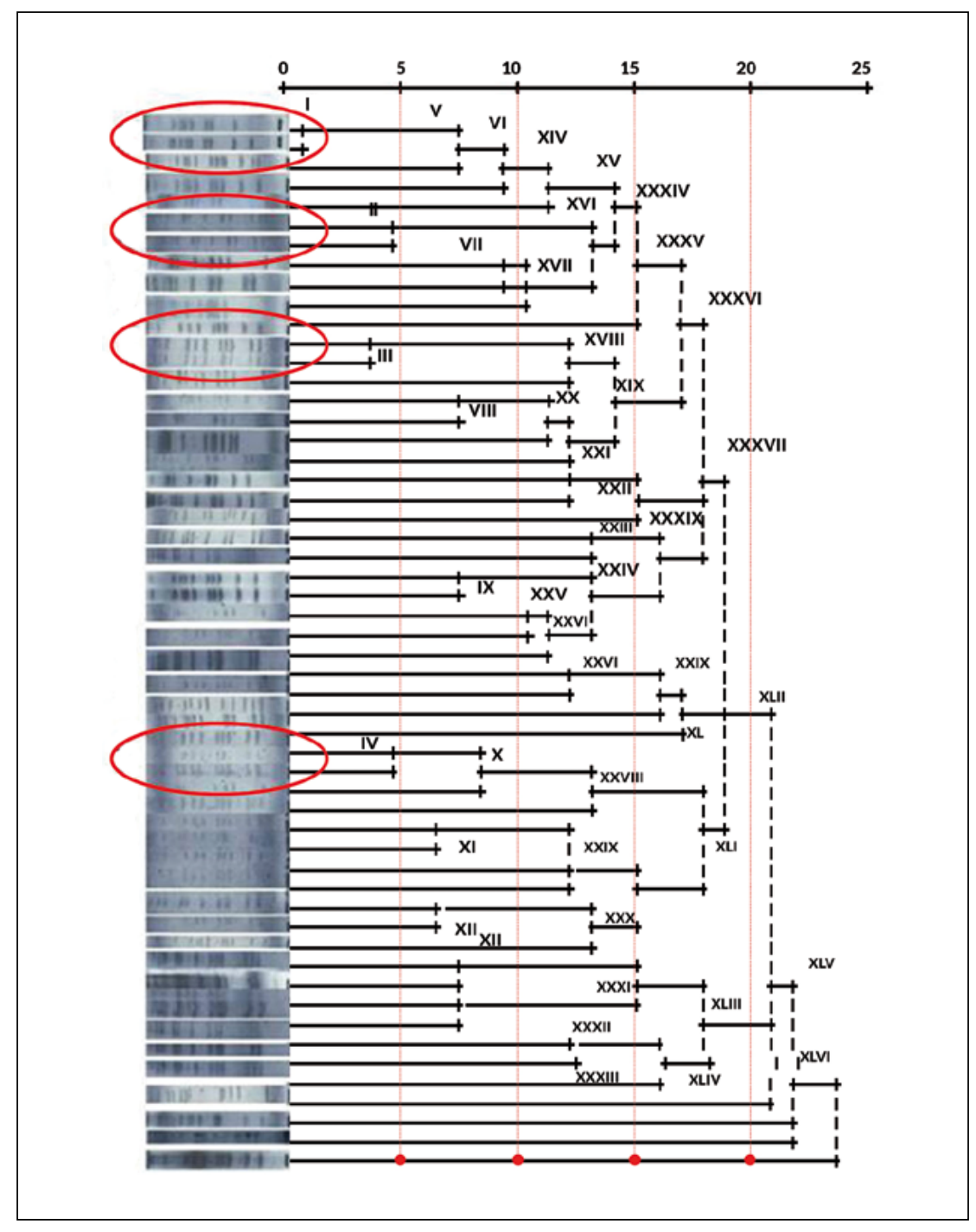

Figura 3. Dendrograma de cepas SARM genotipificadas mediante EGCP.

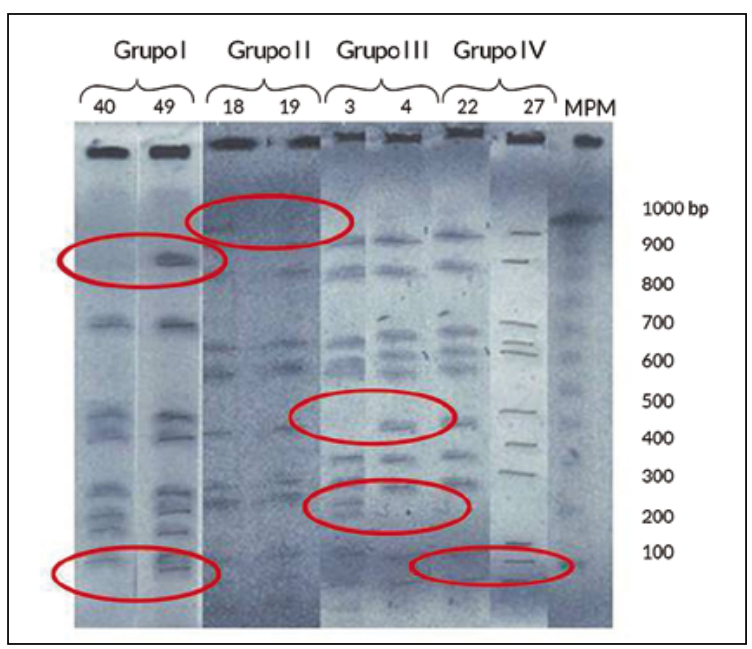

Figura 4. Diferencias observadas entre los pulsotipos encontrados en cepas SARM. 
sistencia de manera homogénea, haciéndose clínicamente relevantes ${ }^{3,28}$. La no detección de estas cepas puede obedecer al empleo de discos de FOX para predecir la resistencia a oxacilina; esta cefamicina ha sido recomendada para las pruebas de resistencia fenotípicas, debido a que es un mejor inductor de $m e c A$ que la $\mathrm{OX}^{3,14,28}$.

Acorde con referencias internacionales, todos los aislados de SARM permanecen susceptibles a QDA, TEC, FUS, LZD, FOS, TGC y MUP $3,4,11,28,29$; por lo que todavía constituyen fármacos importantes para la antibioticoterapia de las infecciones por SARM en la localidad.

SARM expresa resistencia a los antimicrobianos $\beta$-lactámicos que constituyen el tratamiento de elección para las infecciones producidas por $S$. aureus. Además, frecuentemente, expresa resistencia a tres o más grupos de antimicrobianos no relacionados. En esta investigación, con muy pocas excepciones, la mayoría de las cepas fueron multi-resistentes. La determinación de múltiples patrones de resistencia antimicrobiana, indica que carecen de un origen común (al menos recientemente), ya que no responden igual frente a los antimicrobianos ${ }^{11,29-31}$. La multi-resistencia observada constituye una complicación desde el punto de vista del manejo terapéutico adecuado para tratar a los pacientes afectados, así como para controlar su diseminación y evitar brotes de infecciones nosocomiales ${ }^{32}$.

Por décadas, varios métodos fenotípicos y genotípicos han sido utilizados para comprender la diseminación de las cepas de SARM; siendo la EGCP, el método más ampliamente utilizado ${ }^{33}$. Esta técnica basada en la posición de bandas anónimas conlleva algunos inconvenientes, como la falta de relación biológica significativa entre los grupos y las dificultades para la estandarización y comparación entre laboratorios ${ }^{34}$.

Esta dificultad se hizo particularmente evidente, al momento de comparar los resultados aquí obtenidos, con los reportados en la literatura científica internacional, puesto que la tipificación mediante EGCP se efectuó utilizando el equipo GUEFAST, de Neuronic ${ }^{\circledR}$, S.A. y casi toda la literatura disponible, hace referencia es al CHEF-DR II ó III System, (Bio-Rad ${ }^{\circledR}$, Hércules, CA, USA). Sin embargo, puede afirmarse que a pesar que la mayoría de las cepas SARM derivan de un limitado número de clones, ciertas cepas han podido tener un origen policlonal, que sugiere una transferencia horizontal del gen mec $A$ y otros determinantes de resistencia ${ }^{35}$.

Un estudio previo realizado en la institución, demostró la existencia de diseminación clonal de SARM, particularmente en la Unidad de Cuidados Intensivos ${ }^{10}$; no obstante, ahora la situación es completamente diferente, lo que lleva a suponer, que al extremar las medidas de prevención y control de la infección intrahospitalaria, particularmente, el lavado de manos y el uso de guantes, mascarillas y batas desechables, además de controlar la rotación del personal de salud y los traslados de los pacientes a otras áreas del hospital, permitieron en su momento, controlar el brote ocurrente: El resultado es que en la actualidad las cepas de $S$. aureus y en especial, las SARM, provienen de diferentes progenies (origen policlonal), coincidiendo con las afirmaciones de Pérez-Vázquez en España (2009): Este autor no solamente demostró policlonalidad en cepas de SARM, sino también entre cepas SASM, reafirmando la hipótesis de que las cepas SASM provienen de cepas SASM que han adquirido el determinante de resistencia a oxacilina ${ }^{29}$.

Considerando que las cepas fueron obtenidas a principios de 2009, los perfiles observados muestran que los fenómenos genéticos divergentes se produjeron bastante rápido. Esta variabilidad genómica podría explicarse por presiones locales selectivas que fuerzan eventos genéticos recombinantes horizontales, conservando ciertos genotipos variables con éxito. Estudios previos establecían una población altamente clonal, consistente con la opinión de que $S$. aureus, tenía muy pocas probabilidades de recombinarse libremente como otras especies patógenas, tales como: S. pneumoniae, $N$. meningitidis y H. pylori, no siendo naturalmente transformable. En oposición, los resultados obtenidos, indican que SARM posee diversidad genética, en apoyo a las publicaciones de algunos autores, quienes informaron que aunque la recombinación en $S$. aureus es inusual, puede ocurrir dentro y entre loci con suficiente frecuencia para generar un gran número de combinaciones alélicas ${ }^{32,33}$.

Hasta el momento, en Venezuela no se han realizado investigaciones para determinar el (los) clon (es) de SARM circulantes; sin embargo, por cercanía geográfica y por condiciones similares geográficas y económicas, es lógico suponer que en el país, circulen cepas pertenecientes al clon brasileño o al chileno cordobés ${ }^{2}$; afirmación que se ve sustentada por los reportes de caracterizaciones basadas en la susceptibilidad antimicrobiana, que han sido utilizados como una manera económica de fenotipificar las cepas SARM basándose en la susceptibilidad a STX y RA y la multi-resistencia a GM, AK, CC, E, CIP como marcadores fenotípicos para la diferenciación de clones de SARM específicos ${ }^{32,33-35}$. Estas cepas se supone sean portadoras de los SCCmec tipos II y III; aunque estudios realizados por este mismo grupo de investigadores indican que el SCCmec tipo IV también está presente y puede haber adquirido determinantes de resistencia adicionales a los $\beta$-lactámicos y aminoglucósidos, así como los referentes a la resistencia al grupo de macrólidos y lincosamidas. Esta data sugiere que algunas cepas multi-resistentes con el SCCmec IV habrían adquirido tal resistencia para poder sobrevivir en el ambiente hospitalario ${ }^{47}$ o mediante la exposición a dichos antimicrobianos ${ }^{48}$. Tal afirmación encuentra también sustento en la susceptibilidad expresada por las cepas estudiadas a la TE, y que las cepas 
SARM portadoras del SCCmec tipo IV son sensibles a este antimicrobiano ya que carecen de pT181 determinante de la resistencia ${ }^{49}$.

En conclusión, es evidente que existe una alta frecuencia de SARM en la institución de salud estudiada (50,9\%). Estas cepas mostraron fenotipos de multi-resistencia a los antimicrobianos siendo el perfil más común la resistencia simultánea a $\beta$-lactámicos, aminoglucósidos, quinolonas, macrólidos y lincosamidas. Existe un patrón de diseminación policlonal entre las cepas de SARM aisladas en la institución. A pesar de las limitaciones económicas existentes, es pertinente optimizar el uso de los recursos disponibles para continuar investigando la epidemiología de las cepas de SARM en la localidad a fin de poder establecer medidas eficaces tendientes a contener la diseminación de las cepas de SARM multi-resistentes.

\section{Resumen}

Objetivo: Determinar la prevalencia de Staphylococcus aureus resistente a meticilina (SARM), su patrón de susceptibilidad antimicrobiana y tipificar las cepas mediante electroforesis en gel de campo pulsado (EGCP).
Materiales y Métodos: 106 cepas de S. aureus aisladas de pacientes recluidos en un hospital universitario de la ciudad de Maracaibo, Venezuela, fueron procesadas durante el primer trimestre del 2009. El cultivo, aislamiento e identificación de las cepas se hizo por los métodos convencionales. La susceptibilidad antimicrobiana fue determinada por el método de difusión con disco. Se verificó la presencia del gen mec $A$ en las cepas de SARM mediante la reacción de polimerasa en cadena (RPC). $R e$ sultados: Cincuenta y cuatro cepas $(50,9 \%)$ eran SARM y se detectaron veintitrés antibiotipos, siendo el que incluye $\beta$-lactámicos, macrólidos, lincosamidas, aminoglucósidos y quinolonas, el más frecuentemente observado (55,5\%). Hubo cuarenta aislados $(74,0 \%)$ multi-resistentes entre las cepas de SARM. Todas las cepas resistentes a meticilina fueron positivas para mecA. Por EGCP, las cepas de SARM fueron clasificadas en 50 pulsotipos, según el perfil de cortes obtenidos, conteniendo cada uno, entre seis y trece bandas. Cuatro grupos, de dos cepas cada uno, fueron detectados con $80 \%$ de similitud. Cinco de las ocho cepas en estos grupos (62,5\%) tenían el mismo patrón de resistencia. Conclusión: En el hospital, existe una alta prevalencia de cepas SARM multi-resistentes con difusión policlonal.

\section{Referencias bibliográficas}

1.- Cury G, Mobilon C, Guedes E, Lancellotti M, de Carvallo M, Martínez R, et al. Molecular typing of methicillin-resistant Staphylococcus aureus (MRSA) strains isolated in two metropolitan areas of Sao Paulo State, Southeast Brazil. Braz J Infect Dis 2009; 13 (3): 165-9.

2.- Rodríguez Noriega E, Seas C, Guzmán-Blanco M, Mejía C, Álvarez C, Bavestrello L, et al. Evolution of methicillin-resistant Staphylococcus aureus clones in Latin America. Int J Infect Dis 2010;14 (7): e560-6.

3.- Kwai Lin T, Junnie J, Yin Liew F, Yasim Yusof M, Hanifah Y. Antibiograms and molecular subtypes of methicillin-resistant Staphylococcus aureus in local teaching hospital. Malasya J Microbiol Biotechnol 2009; 19 (10): 1265-70.

4.- Campanile F, Bongiorno D, Borbone S, Stefani S. Hospital-associated methicillinresistant Staphylococcus aureus (HA_MRSA) in Italy. Ann Clin Microb Antimicrob; 2009: 8-22.

5.- Bustos-Martínez J, Hamdan-Partida A, Gutiérrez-Cárdenas M. Staphylococcus aureus: la reemergencia de un patógeno en la comunidad. Rev Biomed 2006;17: 287-305.

6.- Vindel A, Cuevas O, Cercenado E, Marcos C, Bautista V, Castellares C, et al. Methicillinresistant Staphylococcus aureus in Spain: molecular epidemiology and utility of different typing methods. J Clin Microbiol 2009; 47 (6):
1620-7.

7.- Larsen A, Goering R, Stegger M, Lindsay J, Gould K, Hinds J, et al. Two distinct clones of methicillin-resistant Staphylococcus aureus (MRSA) with the same USA300 pulsed-field gel electrophoresis profile: a potential pitfall for identification of USA300 communityassociated MRSA. J Clin Microbiol 2009; 47 (11): 3765-8

8.- Cookson B D, Robinson D A, Monk A B, Murchan S, Deplano A, De Ryck R, et al. Evaluation of molecular typing methods in characterizing a European collection of epidemic methicillin-resistant Staphylococcus aureus strains: the HARMONY collection. J Clin Microbiol 2007; 45 (6): 1830-7.

9.- Velázquez-Meza M, Aires De S, Echaniz-Avilés G, Solórzano-Santos F, Miranda-Novales G, Silva-Sánchez J, et al. Surveillance of methicillin-resistant Staphylococcus aureus in a pediatric hospital in Mexico City during a 7-year period (1997 to 2003): clonal evolution and impact of infection control. J Clin Microbiol 2004; 42 (8): 3877-80.

10.- Castellano-González M, Perozo-Mena A, Vivas-Vega R, Ginestre-Pérez M, Rincón-Villalobos G. Tipificación molecular y fenotípica de Staphylococcus aureus resistentes a meticilina (SAMR) en un hospital universitario. Rev. Chilena Infectol 2009; 26 (1): 39-48.

11.- Shittu A, Nubel U, Udo E, Lin J, Gaogakwe S.
Characterization of methicillin-resistant Staphylococcus aureus isolates from hospitals in KwaZulu-Natal Province, Republic of South Africa. J Med Microbiol 2009; 58: 1219-26.

12.- Nubel U, Roumaagnac P, Feldkamp M, Song J, Ko K, Zhuang Y, et al. Frequent emergence and limited geographic dispersal of methicillin-resistant Staphylococcus aureus. Proc Natl Acad Sci USA 2008; 105: 14130-35.

13.- Ministerio del Poder Popular para Ciencia Tecnología. Código de Bioética y Bioseguridad. Caracas, República Bolivariana de Venezuela. 2009.

14.- CLSI. Performance Standards for Antimicrobial Susceptibility Testing. Twentieth Informational Supplement M100-S22. Waynw, PA: Clinical and Laboratory Standards Institute; 2013

15.- Gobernado M. Fosfomicina. Rev Esp Quimio 2003; 16: 15-40.

16.- British Society for Antimicrobials and Chemotherapy (BSAC). BSAC Standardized disc susceptibility method. version 9.1, 1-67. 2010. London, UK, BSAC.

17.- Martineau F, Picard F, Lansac N, Menard C, Roy P, Ouellette M, et al. Correlation between the resistance genotype determined by multiplex PCR assays and the antibiotic susceptibility patterns of Staphylococcus aureus and Staphylococcus epidermidis. Antimicrob Agents Chemother 2000; 44 (2): 231-8.

18.- Backit Neuronic S A. Kit para la obtención 
de ADN genómico de bacterias intacto e inmovilizado. Protocolo. 2007. La Habana Cuba, Neuronic S A.

19.- Sader H. Resistencia antimicrobiana en Latinoamérica: ¿Cómo estamos?. Rev Chilena Infectol 2002; 19 (supl 1) : S5-S13.

20.- Guzmán-Blanco M, Mejía C, Isturiz R, Álvarez C, Bavestrello L, Gotuzzo E, et al. Epidemiology of methicillin-resistant Staphylococcus aureus (MRSA) in Latin America. Int. J. Antimicrob Agents 2009; 34 (4): 304-8.

21.- Pan American Health Organization. Annual Report of the Monitoring/Surveillance Network for Resistance to Antibiotics. Organización Panamericana de la Salud OPS/HDM/ CD/A/408/6; 2004.

22.- Aires de Sousa M, Miragaia M, Sanches I, Avila S, Adamson I, Casagrande S, et al. Three-year assessment of methicillin-resistant Staphylococcus aureus clones in Latin America from 1996 to 1998. J Clin Microbiol 2001; 39 (6): 2197-5.

23.- Echaniz-Avilés G, Velázquez-Meza M, Aires de Sousa M, Morfin-Otero R, RodríguezNoriega E, Carnalla-Barajas N, et al. Molecular characterization of a dominant methicillinresistant Staphylococcus aureus (MRSA) clone in a Mexican hospital (1999-2003). Clin Microbiol Infect 2006; 12 (1): 22-8.

24.- Crisostomo M, Westh H, Tomasz A, Chung M, Oliveira D, de Lencastre H. The evolution of methicillin resistance in Staphylococcus aureus: similarity of genetic backgrounds in historically early methicillin-susceptible and -resistant isolates and contemporary epidemic clones. Proc Natl Acad Sci U S A 2001; 98 (17): 9865-70.

25.- Enright M, Robinson D, Randle G, Feil E, Grundmann H, Spratt B. The evolutionary history of methicillin-resistant Staphylococcus aureus (MRSA). Proc Natl Acad Sci USA 2002; 99 (11): 7687-92.

26.- Castellano González M, Perozo Mena A. Mecanismos de resistencia a antibióticos $\beta$-lactámicos en Staphylococcus aureus. Kasmera 2010; 38 (1): 18-35.

27.- González I, Niebla A, Roura G, González L, Travieso F, Lemus M, et al. Resistencia a las penicilinas en La Habana, Cuba y su incidencia en el género Staphylococcus. Frecuencia de aparición de estafilococos resistentes a meticilina. Rev Panam Infect 2002; 5 (1).

28.- Cuirolo A, Plata K, Rosato A. Development of homogeneous expression of resistance in methicillin-resistant Staphylococcus aureus clinical strains is functionally associated with a beta-lactam-mediated SOS response. J Antimicrob Chemother 2009; 64 (1): 37-45.

29.- Pérez-Vázquez M, Vindel A, Marcos C, Oteo J, Cuevas O, Trincado P, et al. Spread of invasive Spanish Staphylococcus aureus spa-type t067 associated with a high prevalence of the aminoglycoside-modifying enzyme gene ANT(4')-Ia and the efflux pump genes $m s r A$ / $m s r B . J$ Antimicrob Chemother 2009; 63 (1): 21-31.

30.- Strommenger B, Kettlitz C, Werner G, Witte W. Multiplex PCR assay for simultaneous detection of nine clinically relevant antibiotic resistance genes in Staphylococcus aureus. J Clin Microbiol 2003; 41(9): 4089-4094.

31.- Mensa J, García E, Vila J. Macrólidos, cetólidos y estreptograminas. Instituto Clínico de infecciones e inmunología. Hospital Clínico. Barcelona. España. Enferm Infecc Microbiol Clin 2003; 21 (4): 200-8.

32.- Sader H, Jones R N Silva J; SENTRY Participants Group (Latin America).Skin and soft tissue infections in Latin American medical centers: four-year assessment of the pathogen frequency and antimicrobial susceptibility patterns. Diagn Microbiol Infect Dis 2002; 44 (3): 281-8.

33.- Sader H, Jones R, Andrade-Baiocchi S, Biedenbach D; SENTRY Participants Group (Latin America). Four-year evaluation of frequency of occurrence and antimicrobial susceptibility patterns of bacteria from bloodstream infections in Latin American medical centers. Diagn Microbiol Infect Dis 2002; 44 (3): 273-80.

34.- LeMaitre N, Sougakoff W, Masmoudi A, Fievet M H, Bismuth R, Jaruer V. Characterization of gentamicin-susceptible strains of methicillin-resistant Staphylococcus aureus involved in nosocomial spread. J Clin Microbiol 1998; 36: 81-5.

35.- Trzcinski K, Cooper B, Hryniewicz W, Dowson C. Expression of resistance to tetracyclines in strains of methicillin-resistant Staphylococcus aureus. J. Antimicrob. Chemother 2000; 45: 763-70.

36.- Sopena N, Sabriá M. Staphylococcus aureus resistente a la meticilina. Med Clin 2002; 118: 671-76.

37.- Projan S. Antibiotic resistance in the Staphylococci. En: Gram-positive pathogens. Ed. By Fischetti V, Novick R, Ferretti J, Portnoy D, Rood J. Washintong, D.C. United States. American Society for Microbiology Press 2000; pp: 463-70.

38.- Fatholahzadeh B, Emaneini M, Aligholi M, Gilbert G, Taherikalani M, Jonaidi N, et al. Molecular characterization of methicillinresistant Staphylococcus aureus clones from a teaching hospital in Tehran. Jpn J Infect Dis 2009; 62: 309-11.

39.- Ellington M, Ganner M, Warner M, Cookson B, Kearns A. Polyclonal multiply antibiotic-resistant methicillin-resistant Staphylococcus aureus with Panton-Valentine leukocidin in England. J Antimicrob Chemother 2010; 65: 46-50.

40.- Sahida S, Hardy K, Abbasi W, McMurray C, Malik S, Wattal C, et al. Epidemiological typing of methicillin-resistant Staphylococcus aureus isolates from Pakistan and India. J Med Microbiol 2010; 59: 330-7.

41.- Rojas N, Fernández N, Espino M, Fernández M. Patrones de drogorresistencia de cepas de Staphylococcus aureus de origen clínico humano. Rev Cubana Med Trop 2001; 45: 53-8.

42.- Hallin M, Deplano A, Denis O, De Mendonça R, De Ryck R, Struelens M. Validation of pulsed-fiel gel electrophoresis and spa typing for long-term, nationwide epidemiological survillance studies os Staphyloccus aureus infections. J. Clin Microbiol 2007; 45: 127-33.

43.- Lalueza Blanco, A. Importancia actual de la bacteriemia por Staphylococcus aureus en un Hospital Universitario. Tesis Doctoral. Departamento de Medicina. Facultad de Medicina. Universidad Complutense de Madrid. Madrid, España 2008; 191 pp.

44.- Feil E, Holmes E, Bessen D, Chan M, Day N, Enright M, et al. Recombination within natural populations of pathogenic bacteria: short-term empirical estimates and long-term phylogenetic consequences. Proc Natl Acad Sci USA 2001; 98 (1): 182-7.

45.- Amorim M, Faría N, Oliveira D, Vasconcelos C, Cabeda J, Mendes A, et al. Changes in the clonal nature and antibiotic resistance profiles of methicillin-resistant Staphylococcus aureus isolates associated with spread of the EMRSA-15 clone in a tertiary care Portuguese Hospital. J Clin Microbiol 2007; 45: 2881-8.

46.- Nimmo G, Fong J, Paterson D, McLaws M. Changing epidemiology of of methicillinresistant $S$. aureus in Queesland, Australia, 2000-2006: use of a passive surveillance of susceptibility phenotypes. J Hosp Infect 2008; 70: 305-14.

47.- Aires de Sousa M, de Lencastre H. Evolution of sporadic clones of methicillin-resistant Staphylococcus aureus (MRSA) in hospital and their similarities to isolates of communityacquired MRSA. J Clin Microbiol 2003; 41: 3806-15.

48.- Okuma K, Iwakawa K, Turnidge J, Grubb W, Bell J, O’Brien F, et al. Dissemination of new methicillin-resistant Staphylococcus clones in the community. J Clin Microbiol 2002; 40: 4289-94.

49.- Deurenberg R, Kalenic S, Friedrich A, van Tiel F, Stobberingh E. Molecular epidemiology of methicillin-resistant Staphylococcus aureus en Communicating Current Research and Educational Topics and Trends in Applied Microbiology. Méndez-Vilas A (ed). Badajoz: Formatex, USA. 2007; pp: 766-77. 